Article

\title{
Research on the Hydrophobicity of Square Column Structures on Monocrystalline Silicon Fabricated Using Micro-Machining
}

\author{
Ziyang Cao ${ }^{1,2, * \mathbb{D}}$, Wenyu Ding ${ }^{1,2}$, Zhenwu Ma ${ }^{1,2}$, Bangfu Wang ${ }^{1,2}$ and Zhongwang Wang ${ }^{1,2}$ \\ 1 College of Mechanical Engineering, Suzhou University of Science and Technology, Suzhou 215009, China; \\ luckie_d@126.com (W.D.); mazw@usts.edu.cn (Z.M.); sunboy02@126.com (B.W.); \\ maxwang_zw@126.com (Z.W.) \\ 2 Suzhou Key Laboratory of Precision and Efficient Machining Technology, Suzhou 215009, China \\ * Correspondence: dukeczy@nuaa.edu.cn; Tel.: +86-0512-6832-1707
}

Received: 3 October 2019; Accepted: 9 November 2019; Published: 11 November 2019

\begin{abstract}
The theoretical prediction models of contact angle were constructed by considering the interface free energy. Then, the square column structure on monocrystalline silicon was fabricated using micro-milling. The rationality of prediction models was validated by regulating the parameters of the square column. It should be mentioned that the whole construction process was facile and efficient. After processing, the hydrophobicity of monocrystalline silicon with the square column structure was improved. The static contact angle of the processed monocrystalline silicon reached $165.8^{\circ}$ when the side length of the square column was $60 \mu \mathrm{m}$. In addition, the correctness of the prediction models was verified from the perspective of molecular dynamics. The prediction models of contact angle were of great value for the practical application.
\end{abstract}

Keywords: prediction model; square column structure; hydrophobicity; micro-milling; molecular dynamics

\section{Introduction}

In recent years, a great deal of research has been carried out on the hydrophobicity of materials and remarkable progress has been achieved [1]. As a superhydrophobic material, the leg of the water strider has attracted wide attention and been used in various fields in recent decades. A hydrophobic surface refers to a surface that is not easily moistened by water droplets, and its contact angle with water is not less than $90^{\circ}$ [2]. The value of the contact angle can reflect the wettability of a solid surface, i.e., the smaller the contact angle, the better the wettability of the solid surface. Silicon is a key material for microelectronics, optoelectronics, and sensors, and has been widely used in the manufacturing of sensors, solar cells, and many other optical components. The microstructure of the surface of monocrystalline silicon can significantly regulate the hydrophobicity of this material. The existence of hydrophobicity can greatly change the wear resistance and drag reduction of the material and achieve the ability of self-cleaning. When the droplets are placed on a hydrophobic surface, the dust and impurities on the surface will be carried away from the material together with the droplets. Thus, the self-cleaning function of a solar device can be realized by adding a hydrophobic layer [3,4].

Monocrystalline silicon is naturally hydrophobic. These silicon-based hydrophobic surfaces are usually obtained by constructing micro or nanostructures on silicon surfaces to reduce the surface energy. To achieve a higher hydrophobicity, the micro or nanostructure is required for the surface of the silicon-based material [5]. Various approaches have been developed to construct hydrophobic surfaces. Yang et al. [6] and Gurav et al. [7] developed a sol-gel method to prepare new coatings. 
Chen et al. [8] used a special electrodeposition processing method to prepare a superhydrophobic cathode surface on a copper substrate in an electrolytic solution containing $\mathrm{NiCl}_{2} \cdot 6 \mathrm{H}_{2} \mathrm{O}$, tetradecanoic acid and ethanol. Wang et al. [9] adopted an ion etching method to make microstructures for Si. Razavi et al. [10] used chemical vapor deposition to form a microstructure on the surface of copper sheets. Hu et al. [11] used laser to fabricate the titanium alloy to get a biomimetic superhydrophobic surface. Varlamova et al. [12] modified the surface properties of Si using laser-induced periodic surface structures. However, hydrophobic surfaces are mostly constructed using chemical processing and non-traditional processing, and the hydrophobic structure generated by these processing methods is random. In the context of microfabrication, micro-milling technology has gradually developed. Compared with laser processing, Electric discharge machining (EDM) and other micro-machining technologies, micro-milling technology can realize the construction of three-dimensional structures and large-area structure preparation better [13]. The controllability and repeatability of the micro-milling is also good. The structure prepared using micro-milling technology can be well matched with the structural variables of the theoretical model to achieve an accurate exploration of the correlation between the structure and wettability of the surface [14].

Furthermore, theoretical models corresponding to different structures have been established. Marmur et al. [15] analyzed the cylindrical, hemispherical, parabolic and cylindrical protrusions, and constructed the corresponding models. Wang et al. [16] established a theoretical model of an aluminum alloy surface with square column structures that were based on the roughness factor. However, based on the exploration of Gibbs free energy and structural parameters, there are only a few models that explore the hydrophobicity of square column structures or to predict the contact angle of the square column structure visually. Based on Gibbs free energy, Shi et al. [17] constructed prediction models of contact angle for polymethyl methacrylate (PMMA) and $\mathrm{Ti}_{6} \mathrm{Al}_{4} \mathrm{~V}$ with convex structures. However, the square column structures were not mentioned.

In this work, by combining the parameters of micro-structure and interface free energy, reasonable theoretical models were established. The contact angle value could be directly predicted through the parameters of the structure in the models. Furthermore, micro-milling was selected to construct the structure to verify the theoretical models. The whole construction process was facile and efficient, without the requirement of any special devices. The constructed theoretical models could predict static contact angles very well. In addition, experiments showed that excellent hydrophobicity usually occurred when the side length of square column was small. Finally, a simulation of molecular dynamics was carried out to verify the trend of the contact angle at the molecular level. The method of fabricating hydrophobic structure on monocrystalline silicon proposed in this study provides a reference for large-scale industrial production.

\section{Establishment of the Theoretical Model}

\subsection{Basic Parameters of the Model}

Due to the variety of microstructures, existing models are aimed at predicting the contact angle of materials with rough surfaces. It is impossible to visually investigate the influence of microstructure parameters on the hydrophobic properties of materials. Thus, two kinds of models were built based on the wetting state. In this paper, the interface contact lines were straight lines. Then, according to the wetting phenomenon of droplets on the surface of the material, the constructed models were divided into a complete infiltration model and an air column model. Figure 1 shows the two kinds of models. $R$ represents the radius of the contact surface of the droplet with the surface of the material, $a$ represents the side length of square column, and $b$ represents the spacing width of the square column. $h$ is the height of square column, $\rho$ represents the radius of the contact surface between the droplet and the solid. $\theta_{i}$ and $\theta_{a}$ are the contact angles under different wetting models, respectively. $\widetilde{b}$ is the length of the gas-liquid contact line between the square column structures. $A_{s l}$ is the contact area of the 
solid-liquid interfaces, $A_{l v}$ is the contact area of the gas-liquid interfaces, and $A_{s v}$ is the contact area of the solid-gas interfaces.
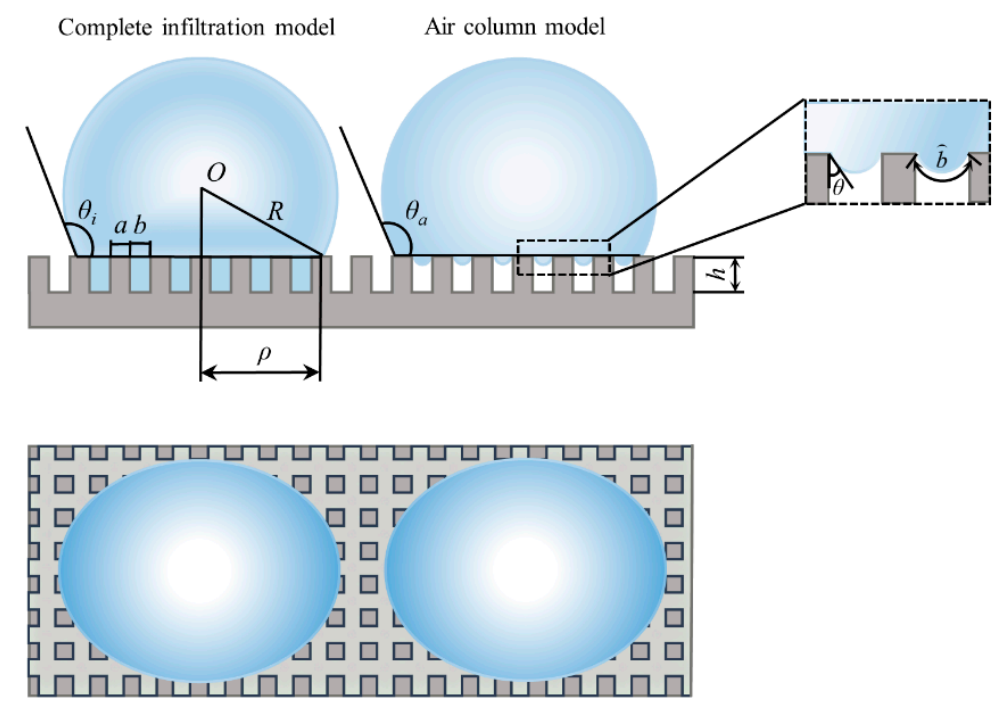

Figure 1. Diagram of the square column model.

The static contact angle was predicted using the model constructed based on the minimum Gibbs free energy. The Gibbs free energy can be obtained using the surface free energy and surface contact area:

$$
G=\gamma_{s l} A_{s l}+\gamma_{l v} A_{l v}+\gamma_{s v} A_{s v}
$$

where $G$ is the Gibbs free energy; $\gamma_{s l}, \gamma_{l v}$, and $\gamma_{s v}$ are the interfacial free energy of the solid-liquid, gas-liquid, and solid-gas interfaces, respectively; and $A_{s l}, A_{l v}$, and $A_{s v}$ are the contact area of the solid-liquid, gas-liquid, and solid-gas interfaces, respectively.

The Gibbs free energy will be minimized when the droplet reaches equilibrium on the surface of the material. Thus, $d G=0$.

The value of surface tension is equal to the value of the surface free energy. By substituting this into Equation (1) and differentiating, this gives:

$$
\sigma_{s l} d A_{s l}+\sigma_{l v} d A_{l v}+\sigma_{s v} d A_{s v}=0
$$

Based on the parameters of the constructed structure, the number of square column structures below the droplet can be expressed as Equation (3):

$$
N=\frac{\pi \rho^{2}}{(2 \rho)^{2}} \cdot\left(\frac{2 \rho}{a+b}\right)^{2}=\frac{\pi \rho^{2}}{(a+b)^{2}} .
$$

\subsection{Establishment of the Square Column Model}

According to the exploration of the square column structure shown in Figure 1, the contact areas between the three phases were represented by the structural parameters, which are shown in Equations (4)-(6), respectively:

$$
\begin{gathered}
A_{s l}=\pi \rho^{2}+N \cdot 4 a h=\pi \rho^{2}+\frac{4 a h \pi \rho^{2}}{(a+b)^{2}}, \\
A_{l v}=\int_{0}^{\theta_{i}} 2 \pi \rho R d \theta=\frac{2 \pi \rho^{2}}{1+\cos \theta_{i}^{\prime}}
\end{gathered}
$$




$$
A_{s v}=0 .
$$

By substituting these contact areas and Equation (2), the droplet balance equation $\sigma_{s v}=\sigma_{s l}+$ $\sigma_{l v} \cos \theta$ can be expressed as Equation (7), where $\theta$ is the intrinsic contact angle of monocrystalline silicon:

$$
\cos \theta=\frac{d A_{l v}}{d A_{s l}}=\frac{(a+b)^{2}}{(a+b)^{2}+4 a h} \cdot \cos \theta_{i}
$$

For the droplet infiltrate structure, the contact angle can be expressed as Equation (8), as follows:

$$
\cos \theta_{i}=\left[1+\frac{4 a h}{(a+b)^{2}}\right] \cos \theta
$$

In contrast, the contact area of the air column model is different from that of complete infiltration model. As shown in Figure 1, it looks like there is air between the square column structure and droplets in the air column model. For the air column model, the surface curvature resulted in an over-pressure in the liquid relative to the exterior pressure $[18,19]$. As a result, the gas-liquid contact line between the structures was not always a straight line.

The length of the gas-liquid contact line between square column structures is:

$$
\widehat{b}=\frac{b}{2 \sin (\theta+\delta)} \cdot(2 \pi-2 \theta-2 \delta),
$$

where $\delta$ is the angle between the square column and the plane. In this study, $\delta=\pi / 2$. The ratio of the gas-liquid contact line length to the spacing width of square column structure is:

$$
\frac{\widehat{b}}{b}=\frac{\frac{b}{2 \cos \theta} \cdot(\pi-2 \theta)}{b}=\frac{\pi-2 \theta}{2 \cos \theta} \approx 1
$$

Thus, $\widehat{b} \approx b$, which means the gas-liquid contact lines between the square column structures were approximately the line segment parallel to the boss.

Combining the wet state of the droplet on the structure and the structural parameters of the microstructure, the contact areas between the three phases were expressed as Equations (11)-(13), respectively:

$$
\begin{gathered}
A_{s l}=N \cdot a^{2}=\frac{a^{2} \pi \rho^{2}}{(a+b)^{2}} \\
A_{l v}=\int_{0}^{\theta_{a}} 2 \pi \rho R d \theta+\pi \rho^{2}-\frac{a^{2} \pi \rho^{2}}{(a+b)^{2}}=\frac{2 \pi \rho^{2}}{1+\cos \theta_{a}}+\pi \rho^{2}-\frac{a^{2} \pi \rho^{2}}{(a+b)^{2}} \\
A_{s v}=A_{\text {total }}-A_{s l}
\end{gathered}
$$

Based on the contact areas and Equation (2), the droplet balance equation can be expressed as Equation (14):

$$
\cos \theta=\frac{d A_{l v}}{d A_{s l}}=\frac{(a+b)^{2}}{a^{2}} \cdot \cos \theta_{a}+\frac{(a+b)^{2}}{a^{2}}-1
$$

The relationship between the contact angle and geometric parameters under the air column model can be obtained using Equation (15):

$$
\cos \theta_{a}=\frac{a^{2}}{(a+b)^{2}}(\cos \theta+1)-1
$$

Through Equations (8) and (15), the correlation between the contact angle and structural parameters were obtained using MATLAB (ver. Matlab 2016, MathWorks, Natick, MA, USA). Figure 2 shows the 
relationship between the contact angle and the structural parameters. According to Figure $2 \mathrm{a}$, it can be seen that as the spacing width of the square column increased, the contact angle of the air column model gradually increased. When $b=300 \mu \mathrm{m}$, the contact angle was larger than for smaller $b$ values. However, the trend of the contact angle predicted using the complete infiltration model was opposite to that of the air column model. Figure $2 b$ shows the curve showing the relationship between the contact angle and the side length of the square column when $b=300 \mu \mathrm{m}$ and $h=100 \mu \mathrm{m}$.

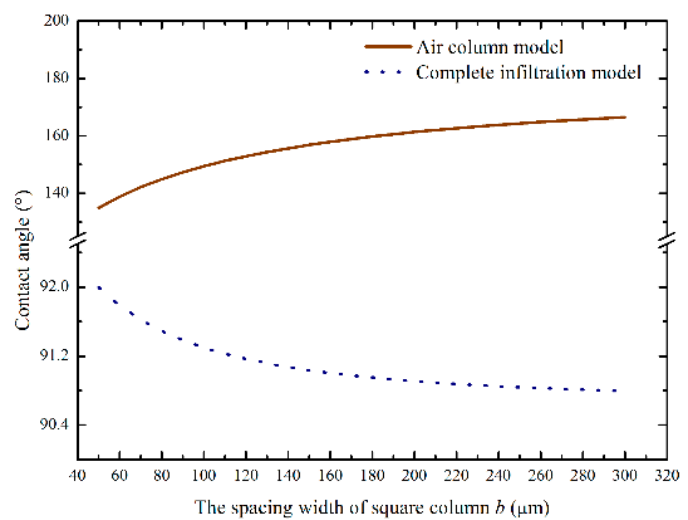

(a)

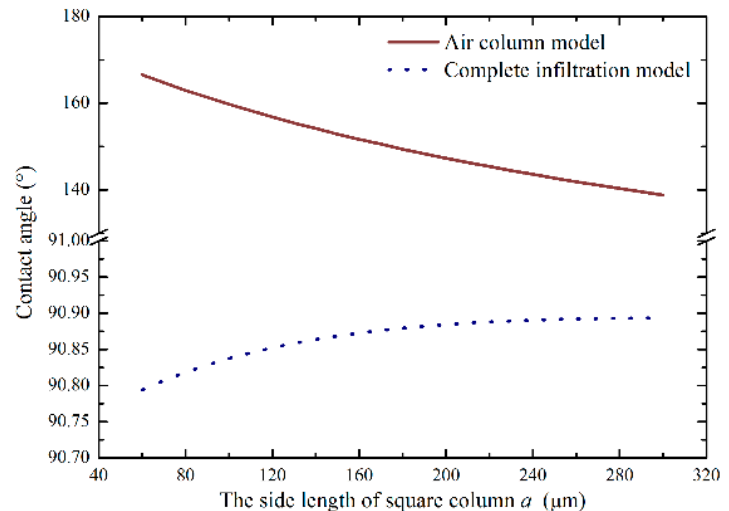

(b)

Figure 2. Correlation between the contact angle and structural parameters: (a) the spacing width of square column and $(\mathbf{b})$ the side length of the square column.

From Figure 2b, it can be seen that the changes in structural parameters had an effect on the hydrophobicity of monocrystalline silicon containing microstructures. The hydrophobicity of the air column model gradually increased as the length of the square column decreased. In contrast, the contact angle predicted using the complete infiltration model was larger as the side length of the square column increased.

\section{Experimental and Simulation Verification}

\subsection{Experimental Verification}

Experiments were carried out to verify the contact angle predicted by the prediction models. In this paper, the experimental samples were monocrystalline silicon wafers $\left(\begin{array}{ll}1 & 0\end{array}\right)$, Shunsheng, Ningbo, China). According to the physical properties of the monocrystalline silicon, 100 crystal planes were selected and the surface was polished. The diameter parameter of these samples was $25 \mathrm{~mm}$, and the thickness was $400 \mu \mathrm{m}[20,21]$. All of these monocrystalline silicon samples were cleaned using an ultrasonic cleaner (JP-010T, Skymen, Shenzhen China) containing ethanol and a 10\% HF aqueous treatment to remove the burrs and oxide grown on the surface of the samples. Then, the metallographic tapes were stuck on the surface of these nitrogen-dried samples to isolate it from the air, ensuring accurate measurements and processing. The workpieces were machined using a double-edged micro-milling cutter (NTS-Cutting, Natasi, Dongguan China). The machining system used in this paper was a high-speed milling center. The tool selected was a diamond coated micro-milling cutter with a diameter of $0.2 \mathrm{~mm}$. The spindle speed was $48,000 \mathrm{r} / \mathrm{min}$ and the feed speed was $6 \mathrm{~mm} / \mathrm{min}$ during experiments [22]. Six specimens were processed with 15 grooves distributed on the intermediate surface of the workpieces to form a square column structure.

All processed samples were cleaned with an ethanol-containing ultrasonic cleaner for $5 \mathrm{~min}$ to remove the burrs and impurities remaining on the surface and between the structures of the monocrystalline silicon. After the impurities were cleaned, the prepared 10\% HF aqueous solution was placed in the ultrasonic cleaner to remove the oxide grown on the surface of samples. Finally, the surface of the specimens was dried using nitrogen for a follow-up analysis. The apparent morphology of 
samples was observed with a VHX-5000 Superhigh magnification lens zoom 3D microscope (VHX-5000, Keyence, Osaka, Japan). The static contact angles of these specimens were measured using an optical contact angle measuring instrument (OCA, DataPhysics, Filderstadt, Germany). It was necessary to measure the contact angle of each specimen five times to ensure the reliability of the measured data. Furthermore, a fixed area was selected as the measuring point of the contact angle to ensure accurate experimental data. The processing flow chart for creating monocrystalline silicon with square column structures is shown in Figure 3.

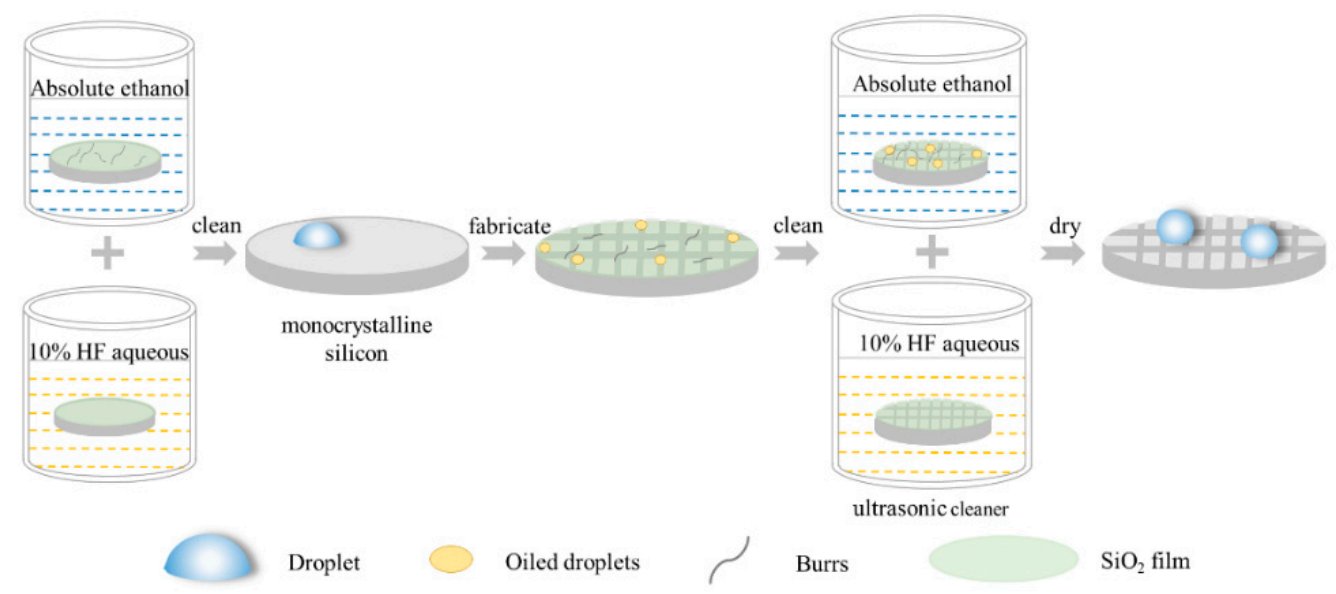

Figure 3. The processing flow chart for monocrystalline silicon.

The purpose of this experiment was to verify the hydrophobicity of silicon surfaces containing square column microstructures by changing the side length of the square column.

In the experiment, the side length of the square column $a$ was a variable, while the spacing width $b$ and the height $h$ of the square column were constant, where $b=300 \mu \mathrm{m}$ and $h=100 \mu \mathrm{m}$.

\subsection{Simulation Verification}

The state of the water droplets with different geometric surfaces was studied from the perspective of molecular dynamics. In this paper, the droplets were simulated using an SPC water model. The water-water and Si-water interactions in this paper were described using the Lennard-Jones (LJ) potential [23]:

$$
\cos \theta_{a}=\frac{a^{2}}{(a+b)^{2}}(\cos \theta+1)-1
$$

The Si-water potential was obtained using the Lorentz-Berthelot rule:

$$
\begin{gathered}
\varepsilon_{\mathrm{sw}}=\sqrt{\varepsilon_{s} \times \varepsilon_{w}} \\
\sigma_{\mathrm{sw}}=\frac{\sigma_{s}+\sigma_{w}}{2}
\end{gathered}
$$

The simulation was carried out at $298 \mathrm{~K}$. The Nose-Hoover temperature coupling algorithm was adopted in this paper. Figure 4 shows the schematics of the simulation model. The square column structure was obtained by removing the cells of the smooth structure [24]. 

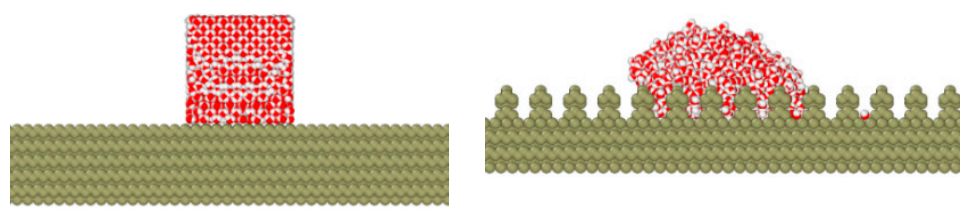

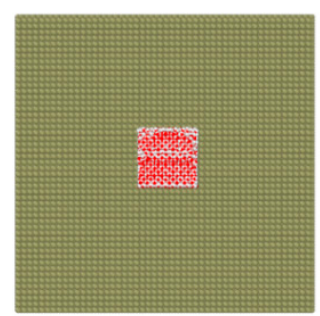

(a)

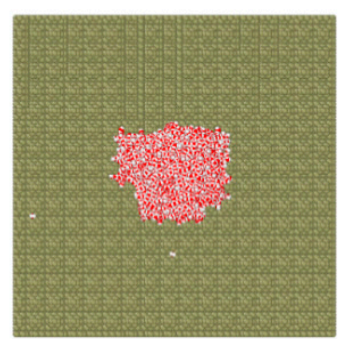

(b)

Figure 4. The schematics of the simulation model: (a) initial model and (b) model after simulation.

Figure 5 shows the density profile of the water droplet. In the density profile, the edge contour was extracted and the contact angle was derived at the solid-liquid boundary.

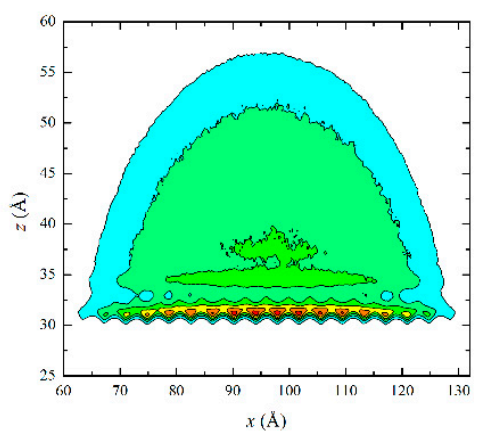

(a)

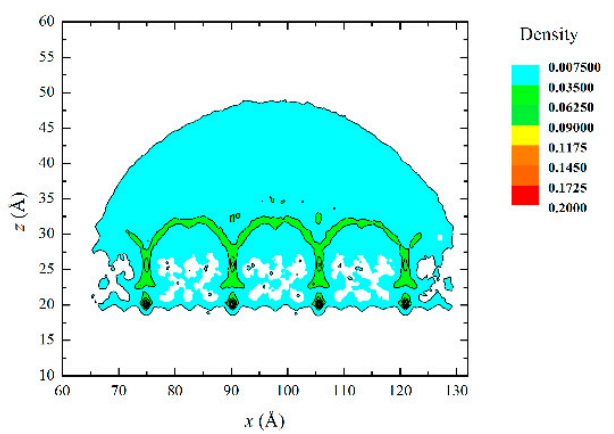

(b)

Figure 5. Density profile of a water droplet: (a) initial model and (b) model after simulation.

\section{Results and Discussion}

According to multiple measurements, the average contact angle of the unprocessed monocrystalline silicon was $90.67^{\circ}$. The monocrystalline silicon was naturally hydrophobic. Figure 6 shows the structure diagram of the samples after machining. The theoretical value of the contact angle was calculated according to Equations (8) and (15). The experimentally measured value and predicted value of the contact angle are shown in Table 1. Figure 7 is plotted based on the data in Table 1. 


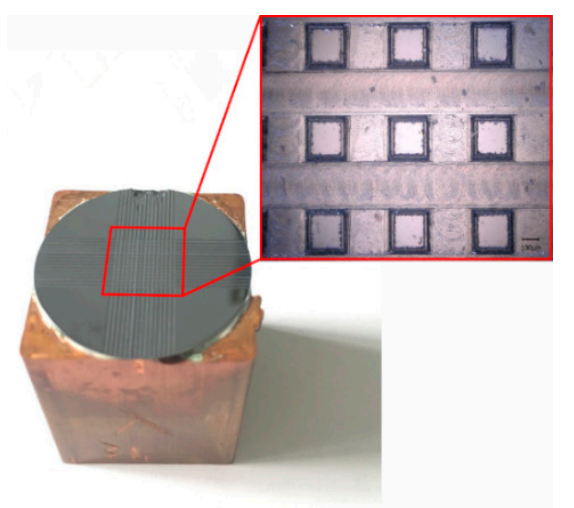

Figure 6. Surface diagram of the processed square column structure.

Table 1. The theoretical and experimental values of contact angle.

\begin{tabular}{ccccc}
\hline Sample Number & $\begin{array}{c}\text { Side Length of } \\
\text { Square Column } \boldsymbol{a} \\
(\boldsymbol{\mu} \mathbf{m})\end{array}$ & $\begin{array}{c}\text { Complete } \\
\text { Infiltration Model } \\
\boldsymbol{\theta}_{\boldsymbol{i}}\left(^{\circ}\right)\end{array}$ & $\begin{array}{c}\text { Air Column } \\
\text { Model } \boldsymbol{\theta}_{\boldsymbol{a}}\left({ }^{\circ}\right)\end{array}$ & Experiment $\boldsymbol{\theta}\left({ }^{\circ}\right)$ \\
\hline 1 & 60 & 90.79 & 166.54 & $165.8 \pm 2.2$ \\
2 & 90 & 90.83 & 161.33 & $160.1 \pm 2.1$ \\
3 & 120 & 90.85 & 156.83 & $158.6 \pm 1.8$ \\
4 & 150 & 90.87 & 152.89 & $156.7 \pm 3.0$ \\
5 & 180 & 90.88 & 149.43 & $149.7 \pm 2.5$ \\
6 & 200 & 90.88 & 147.34 & $146.3 \pm 2.6$ \\
\hline
\end{tabular}

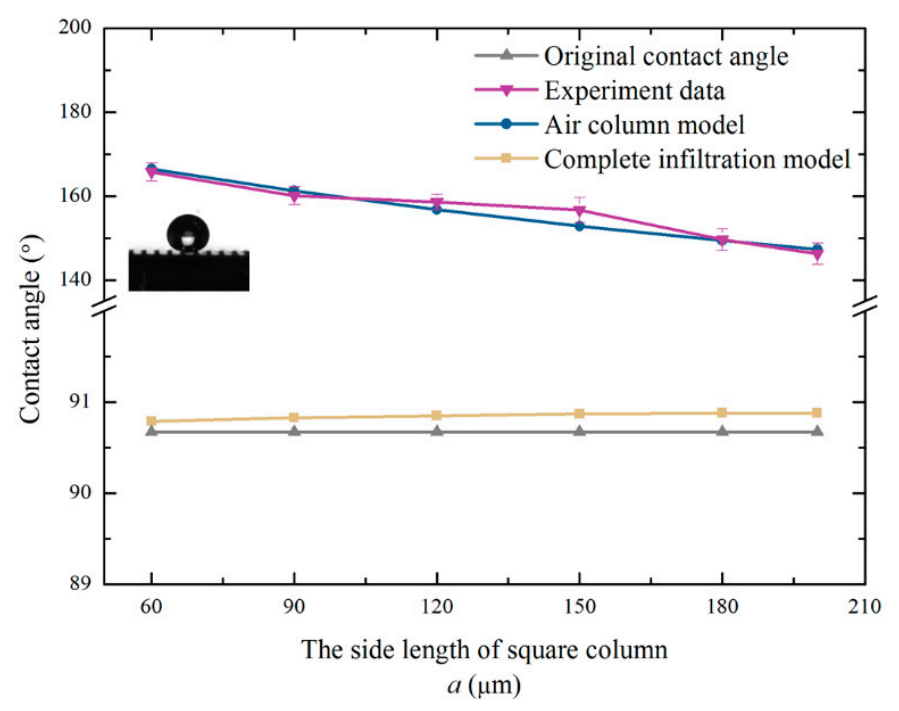

Figure 7. Variation curve of the contact angle between measurement and theory.

It is clear that the contact angles of the six specimens were all improved significantly. The hydrophobicity of the square column structure was dramatic. Furthermore, the air column model was more reliable than the complete infiltration model. Since the solid, liquid, and gas phases were in equilibrium, air was always present below the droplets. The boss constrained the droplet from all directions. Due to the high interfacial tension between the solid and liquid, the droplets were constrained, which caused an increase in the contact angle. The fluctuation between the predicted value and the measured value of the contact angle was very small. The reasonability of the prediction models was verified via the experiments.

According to Figure 8, it can be seen that it was the cracking during processing that caused the slight fluctuation of the contact angle. Monocrystalline silicon is easier to curl during the milling 
process. Due to the large tensile stress of monocrystalline silicon, cracks and breakages occurred easily such that the measured contact angle was slightly larger than the theoretical value. Overall, the prediction models could calculate the contact angle of the square column accurately.
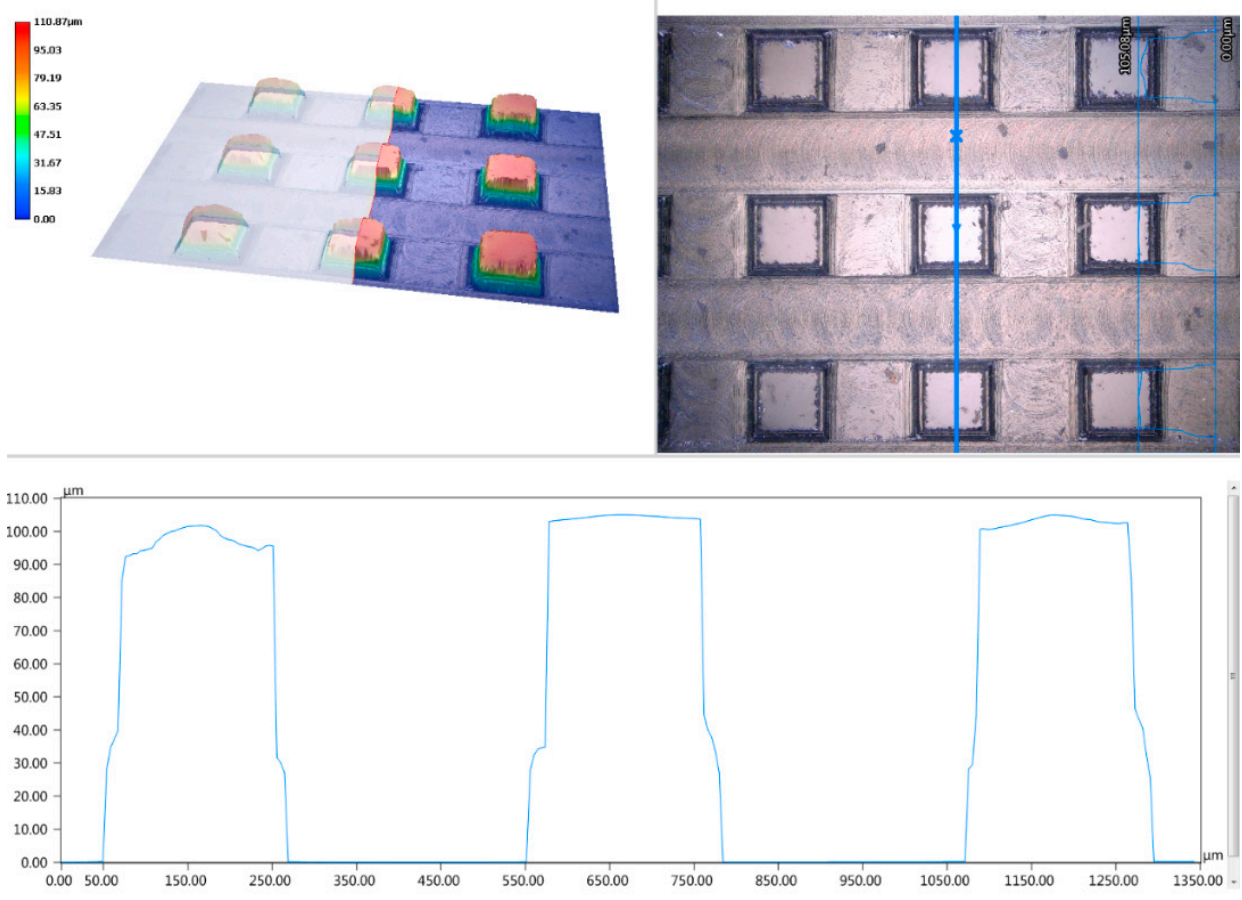

Figure 8. 3D microstructure topography of the square column.

Figure 9 shows the contrast curve of the trend of the simulation data and experimental data. $L_{C}$ is the lattice constant of monocrystalline silicon. It can be seen that the trend of the simulated and measured values of the contact angle are the same. This indicates the correctness and reliability of the prediction model. It is clear that a smaller side length of the square column resulted in better hydrophobicity of solid surface in practical applications. The prediction models played a guiding role on the construction of the hydrophobic surface of monocrystalline silicon.
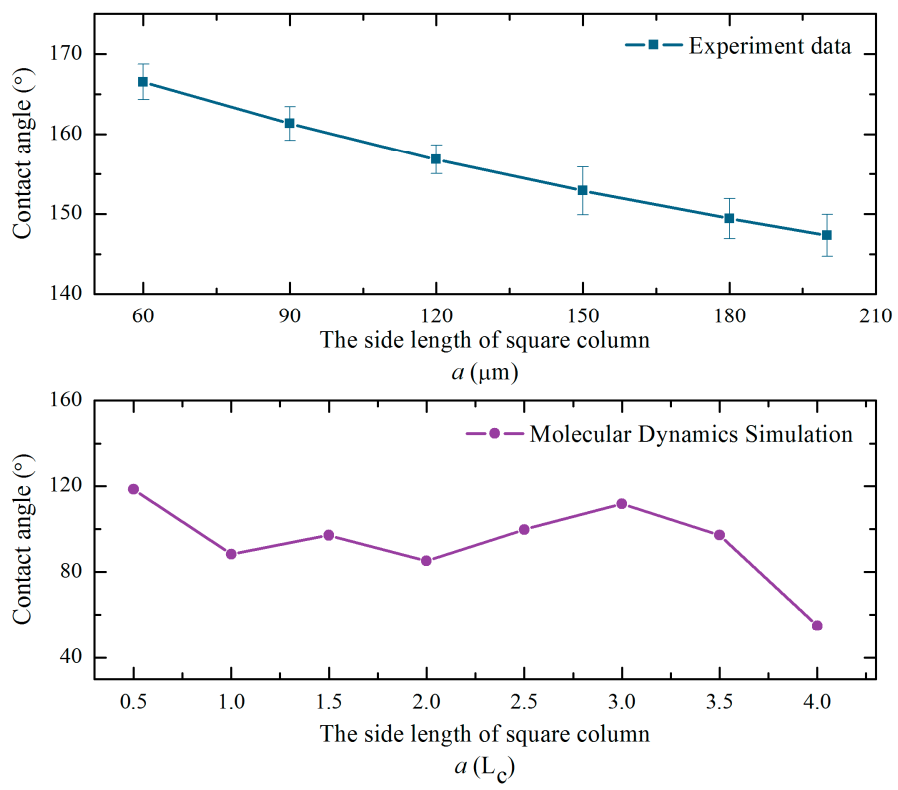

Figure 9. Variation curves of simulated and measured contact angle. 


\section{Conclusions}

In this paper, the prediction models of contact angle were constructed by considering the interface free energy on the basis of micro-milling. Owing to the square column structure constructed using micro-milling, the surface could achieve a stable and effective hydrophobicity. There was a great match between experimental values and theoretical values, which proved the correctness and rationality of the prediction model. Particularly, the geometric parameters of the structure had a great influence on the contact angle. The smaller the side length of the square column, the larger the contact angle was, and the stronger the hydrophobicity of the material. Furthermore, the rationality of the experimental data trend was verified at the level of molecular dynamics, which ensured the correctness of the prediction model. Overall, it was clear that the prediction models played a guiding role in the construction of the hydrophobic surface on monocrystalline silicon. This study provided a reference for the large-scale industrial production of monocrystalline silicon with hydrophobic surface.

Author Contributions: Conceptualization, W.D. and Z.C.; methodology, W.D. and B.W.; software, Z.W. and Z.M.; validation, Z.C.; formal analysis, B.W.; investigation, Z.W.; resources, B. and Z.M.; data curation, B.W.; writing-original draft preparation, W.D.; writing-review and editing, W.D. and Z.C.; visualization, W.D.; supervision, Z.C.; project administration, Z.C.; funding acquisition, Z.C.

Funding: The authors would like to acknowledge the support from the National Natural Science Foundation of China, "Research on the dynamics and stability in high-precision micro-milling of microstructures" (51305286), and the support from the Joint research project situating on the Sino-foreign cooperative education platform in Jiangsu province, "Key technology and equipment for high-efficiency and high finishing of complex surfaces of difficult-to-process material molds."

Acknowledgments: The authors acknowledge the technical staff of our research laboratory for their assistance in carrying out the experiments.

Conflicts of Interest: The authors declare no conflict of interest.

\section{References}

1. Yan, Y.Y.; Gao, N.; Barthlott, W. Mimicking natural superhydrophobic surfaces and grasping the wetting process: A review on recent progress in preparing superhydrophobic surfaces. Adv. Colloid Interface Sci. 2011, 169, 80-105. [CrossRef] [PubMed]

2. Cui, C.; Duan, X.L.; Collier, B.; Poduska, K.M. Fabrication and wettability analysis of hydrophobic stainless steel surfaces with microscale structures from nanosecond laser machining. J. Micro Nano-Manuf. 2018, 6, 031006. [CrossRef]

3. Latthe, S.S.; Sutar, R.S.; Kodag, V.S.; Bhosale, A.K.; Kumar, A.M.; Sadasivuni, K.K.; Xing, R.; Liu, S. Self-cleaning superhydrophobic coatings: Potential industrial applications. Prog. Org. Coat. 2019, 128, 52-58. [CrossRef]

4. Kumar, K.D.; Avramova, I.A.; Castano, C.E.; Ivanova, I.A.; Mohammadi, R.; Radeva, E.I.; Stoyanovad, D.S.; Vladkovae, T.G. Early stage anti-bioadhesion behavior of superhydrophobic soot based coatings towards Pseudomonas putida. Mater. Des. 2018, 160, 395-404.

5. Zorba, V.; Stratakis, E.; Barberoglou, M.; Spanakis, E.; Tzanetakis, P.; Fotakis, C. Tailoring the wetting response of silicon surfaces via fs laser structuring. Appl. Phys. A Mater. Sci. Process. 2008, 93, 819-825. [CrossRef]

6. Yang, H.; Pi, P.H.; Cai, Z.Q.; Wen, X.; Wang, X.; Cheng, J.; Yang, Z.R. Facile preparation of super-hydrophobic and superoleophilic silica film on stainless steel mesh via sol-gel process. Appl. Surf. Sci. 2010, 256, 4095-4102. [CrossRef]

7. Gurav, A.B.; Latthe, S.S.; Kappenstein, C.; Mukherjee, S.K.; Rao, A.V.; Vhatkar, R.S. Porous water repellent Silica coatings on glass by sol-gel method. J. Porous Mater. 2011, 18, 361-367. [CrossRef]

8. Chen, Z.; Hao, L.M.; Chen, A.Q.; Song, Q.; Chen, C. A rapid one-step process for fabrication of superhydrophobic surface by electrodeposition method. Electrochim. Acta 2012, 59, 168-171. [CrossRef]

9. Wang, J.D.; Liu, F.B.; Chen, H.S.; Chen, D. Superhydrophobic behavior achieved from hydrophilic surfaces. Appl. Phys. Lett. 2009, 95, 84-104. [CrossRef] 
10. Razavi, S.M.; Oh, J.; Sett, S.; Feng, L.; Yan, X.; Hoque, M.J.; Liu, A.; Haasch, R.T.; Masoomi, O.M.; Bagheri, R.; et al. Superhydrophobic surfaces made from naturally derived hydrophobic materials. ACS Sustain. Chem. Eng. 2017, 5, 11362-11370. [CrossRef]

11. Hu, L.; Zhang, L.; Wang, D.; Lin, X.; Chen, Y. Fabrication of biomimetic superhydrophobic surface based on nanosecond laser-treated titanium alloy surface and organic polysilazane composite coating. Colloids Surf. A 2018, 555, 515-524. [CrossRef]

12. Varlamova, O.; Hoefner, K.; Ratzke, M.; Reif, J.; Sarker, D. Modification of surface properties of solids by femtosecond LIPSS writing: Comparative studies on silicon and stainless steel. Appl. Phys. A 2017, 123, 725. [CrossRef]

13. Cao, Z.Y.; Ding, W.Y.; Yin, Z. Research on surface integrity analysis and control technology of high precision milling. J. Adv. Oxid. Technol. 2018, 21, 201801239.

14. Ding, W.Y.; Cao, Z.Y.; Wang, B.F.; Xu, S.; Wang, Z. Construction of grating structure model based on Gibbs free energy and experimental verification by micro-milling. Appl. Phys. A 2019, 125, 380. [CrossRef]

15. Bittoun, E.; Marmur, A. Optimizing super-hydrophobic surfaces: Criteria for comparison of surface topographies. J. Adhes. Sci. Technol. 2009, 23, 401-411. [CrossRef]

16. Wang, Y.L.; Zhang, X.R.; Zhang, L.X.; Yu, Z.J. Hydrophobic aluminum alloy surface fabricated by high speed micro-milling technology. China Surf. Eng. 2013, 26, 37-42.

17. Shi, Z.Y.; Liu, Z.Q.; Song, H.; Zhang, Z. Prediction of contact angle for hydrophobic surface fabricated with micro-machining based on minimum Gibbs free energy. Appl. Surf. Sci. 2016, 364, 597-603.

18. Nosonovsky, M.; Bhushan, B. Hierarchical roughness optimization for biomimetic super-hydrophobic surfaces. Ultramicroscopy. 2007, 107, 969-979. [CrossRef] [PubMed]

19. Liu, J.L.; Feng, X.Q.; Wang, G.F.; Yu, S.W. Mechanisms of superhydrophobicity on hydrophilic substrates. J. Phys. Condens. Matter. 2007, 19, 1-12. [CrossRef]

20. Cheng, X.; Gao, B.; Yang, X.H.; Liu, J.Y.; Tian, Z.Q. Experimental study on the ductile-mode micro milling of single crystalline silicon. Shandong Univ. Technol. (Nat. Sci. Ed.) 2012, 26, 53-55.

21. Zhang, S. Design and Development of a Micromilling Machine Tool and Experimental Study on Micromilling of the Typical Hard and Brittle Material. Master's Thesis, Shangdong University of Technology, Zibo, China, June 2014 .

22. Gao, Q.; Gong, Y.D.; Cai, M.; Zhou, Y.G. Experimental study on surface quality in micro-milling of single crystal Si plastic removal. Technol. Test. 2016, 6, 135-138.

23. Yuan, C.S. Investigation of Micro and Nano Structure on Titanium Alloy Surface Influences its Superhydrophobicity. Master's Thesis, Harbin Institute of Technology, Harbin, China, June 2010.

24. Kwon, T.W.; Jang, J.; Ambrosia, M.S.; Ha, M.Y. Molecular dynamics study on the hydrophobicity of a surface patterned with hierarchical nanotextures. Colloids Surf. A 2018, 559, 209-217. [CrossRef] 